\title{
TUBERCULOSIS AND SYPHILIS
}

\author{
By H. C. G. SEMON
}

THAT a man who has syphilis cannot contract a luetic primary chancre is self-evident. An identical stipulation applies to tuberculosis, and as nearly everybody is mildly infected in infancy or early childhood, explains the extreme rarity of the primary tuberculous chancre. In direct contrast, therefore, to what pertains in syphilis, it is exceptional for the T.B. chancre to occur in the adult. Certainly I have never seen such a case myself, and when the possibility is suspected it should always be subjected to the most stringent criticism.

An example that fulfilled all criteria was published some years ago in the Dermatologische Wochenschrift. A young woman, a domestic servant, who was "walking out " with a sailor developed a sore on the chin close to the labial commissure, about three weeks after her fiancé had returned to his ship. Tests for lues having proved negative, both in the exuding serum and in the homolateral adenitis which was associated with the primary lesion, Koch's bacillus was envisaged as a possible factor and recovered in scrapings. Subsequently the glands softened and broke down and tubercle bacilli were found in the secretion. To complete the clinical picture, a patch of lupus vulgaris later made its appearance in the neighbourhood of the chancre, and the source of the infection seemed proven, when the young man reported sick on his next leave and was found to be suffering from laryngeal tuberculosis. It is interesting to note that the incubation period of three weeks in this case coincides very well with that observed in cases of tuberculosis of the frenum 11 and penis in infants, following ritual circumcision, in which the operator-a phthisical rabbi-was known to have staunched, or attempted to staunch, hæmorrhage by oral suction.

Another recorded site for the primary lesion is the vulva in the young child when propulsory sliding efforts are being made on a floor previously contaminated by 
infected sputum. The propensity of infants and young children to put toys in their mouths accounts for the occasional infections reported in this locality, and the possibility of the so-called " tuberculous primary complex" should always be borne in mind when a sore and glandular enlargement of some weeks' duration is presented for an opinion. The "chancre" differs from that of lues in several particulars. The infiltration is softer and the edges are flat and somewhat overhanging, while the base has a rather granular appearance and tends to bleed more easily. The infected glands may be hard at first, but rarely of that rubbery consistency so typical of the venereal adenitis. They may be painless and discreet too at an early stage, but they soon become adherent to each other, soften, and break down with the characteristic involvement of the skin. Typical apple-jelly nodules of lupus may develop both in the neighbourhocd of the sore or close to a sinus discharging pus from caseous glands. It is a sine qua non that no case could be regarded as proved without the microscopic demonstration of the tubercle bacillus, just as no modern syphilologist would ignore the proof that the dark-ground demonstration of the treponema can afford.

The difference in the pathogenesis of the two diseases would seem, then, if traced back to ultimate causes, to depend on the fact that tuberculosis in the large majority of cases is an air-borne infection and can invade the lungs either directly or through the bronchial lymphatics and glands, and in the food through the alimentary tracttwo portals which, because it is so much more vulnerable, and never air-borne, are permanently closed to the luetic infection. That Koch's bacillus most usually enters by one or other is now generally accepted. It must be similarly conceded that the ensuing infection of the viscera is at first silent or latent in so far as clinical symptoms are concerned, but demonstrable and commonplace in view of the frequency with which skin tests prove positive in apparently healthy individuals. From one aspect this silent infection must be regarded as salutary, for without it, considering how universally distributed the fomites are, primary cutaneous tuberculosis with its more dangerous consequences would be more common than it is. That nevertheless a tuberculous subject can contract a tuberculous skin affection, either from his own infection or I6o 
from an extraneous source, cannot be denied. I have observed it in the form of a verrucose tuberculosis of the fingers in old standing pulmonary infections, probably from a contaminated handkerchief, and it is a sign of evil augury when a lung case develops a peri-anal "superinfection" (probably from swallowed sputum), as such examples are rightly called.

Jadassohn regards lupus vulgaris as an invariable super-infection, i.e., that it can only occur in a person already tuberculous, and it is demonstrable that the distinction is applicable to other tuberculous manifestations on the skin. In syphilis the term appears to be used in a more exact sense in relation to the coincidence of two or more primary chancres. The local spread or extension of a tuberculous process is mainly by way of the lymphatics, and in this respect would seem to differ from an otherwise analogous syphilitic lesion. An interesting example of this discrepancy is afforded by what usually happens when the alæ nasi are affected. In lupus the infection is at first localised to the nasal mucosa, but secondary nodules may and often do appear on the skin of the cheeks in the form of lupus patches at any time subsequently. In the analogous tertiary syphilitic lesion the treponemata, if they produce a gummatous infiltration and necrosis, spready by contiguity and are not localised to the lymphatic path. Hence we never see isolated gummatous patches on the cheeks as a secondary extension from a nasal focus of the disease. But this is not to say that cutaneous tuberculosis is not blood-borne. Miliary tuberculosis comes under this heading, and although it rarely attacks the skin in the adult, can certainly produce embolism of the skin in infants and young children. Post-exanthematic lupus in young children is another example of undoubted circulatory transport. It not infrequently occurs in the form of numerous widely disseminated patches on the trunk after recovery from measles, or some acute specific fever, and is considered by Volk, Mibelli and v. Pirquet to be the result of a temporary failure of antibody production during the febrile period. The latter observer found that the previously positive Mantou test became negative during that time. but was again obtainable when the patient had recovered. The observation is of value and goes to support the contention by these authors that during the measles infection I6I 
the child is temporarily deprived of the T.B. antibody protection stored up in previous months or years. It is interesting to compare it with what is supposed to happen when we infect an early G.P.I. with malaria. Here we speak of a mobilisation of antibody production to explain the therapeutic effect and the conversion of a positive W.R. to a negative. By and large it would appear that while intercurrent febrile reactions are harmful in chronic tuberculosis, they are undoubtedly, and in given circumstances, useful and even indicated in the treatment of otherwise intractable syphilitic infection. The effect of intercurrent disease on these two infections is to some extent paralleled by their reactions to pregnancy. It is notorious that a woman with pulmonary tuberculosis runs considerable risks during the periods of pregnancy and lactation, although the chances are that she will give birth to a healthy child. The issues are reversed in the case of syphilis, for in this disease active symptoms either involute or are entirely suppressed, a positive W.R. frequently becomes temporarily negative, while the child may be born with or may shortly develop manifest symptoms of congenital lues. These are facts which no one disputes, but their true explanation is likely to remain obscure and the subject of theory and discussion for years to come.

The clinical differentiation of the cutaneous manifestations of the two infections, which is my thesis in this paper, should of course be based on the underlying histopathology. But nowadays it is commonly omitted, either because few of us are competent in the finer details of their comparative reactions, i.e., their histology, or because the W.R. is a much more rapid, and at least as reliable, discriminatory test.

Speaking broadly, the histological differentiation of tubercular from tertiary syphilitic lesions is based on the fact that tubercles due to Koch's bacillus are more frequently multiple, while the gumma is usually a solitary infiltration with a strong tendency to necrose in its centre. Giant cells may occur in both, but in tuberculosis they have a more typical appearance and arrangement, most characteristically seen in lupus vulgaris, which, once memorised, could hardly be mistaken for any other granuloma. The lupus nodule is a collection of tubercles, composed for the most part of so called epithelioid cells aggregated in multiple groups, in which not infrequently 


\section{TUBERCULOSIS AND SYPHILIS}

actual tubercle bacilli, the cause of their proliferation, can be stained and demonstrated. The giant cells of such tubercles contain nuclei arranged like a horseshoe in the periphery, in contra-distinction to those seen in syphilis and other granulomatous infiltrations in which no such uniformity is demonstrable. The coalescence of several small groups of tubercles to form one or two large ones affords an explanation of the relatively common tuberculous lesion with a polycyclic border with multiple points of caseation, as against the gumma which has a round or oval form and a tendency to central necrosis. The very large number of tuberculides-which include T.B. verrucosis, scrofuloderma, miliary T.B. cutis, lichen scrofulosorum, papulonecrotic tuberculides, Darier's and Boeck's sarcoids, angiolupoid and many other less determined forms-preclude a detailed account of their various deviations from the typical tubercle. On the whole, however, it may be asserted that the resemblances to the trained eye are distinct and rarely liable to confusion with the histology of clinically similar syphilides.

These considerations embolden me to tackle my task in a rather unorthodox manner, and I propose to discuss the subject according to the regional areas which may be involved by the manifestations of the two diseases in late or early stages of their incidence.

\section{(I) The Scalp}

If we except lupus erythematosus, which as you may know is thought to be of tuberculous origin by some authorities, tuberculosis manifests itself but rarely in this situation, so rarely that a case of lupus vulgaris capitis is a curiosity. Gumma of the scalp, on the other hand, would be a commonplace but for salvarsan. Its ravages before the war not infrequently involved the outer table of the skull, and patients sometimes succumbed to a septic meningitis. We are rarely on debatable ground moreover when we are consulted for loss of hair. The moth-eaten appearances of the luetic secondary stage, with or without roseolar rash, are never seen in diffuse fall of hair due to tuberculosis. I remember being conculted for this symptom by a former house physician who died some months later of acute tuberculosis involving both her lungs, infection from which had doubtless been 
the cause, entirely unsuspected at the time, of her alopecia. The cicatricial localised types of follicular infection are rarely attributable to either disease, and although it is always advisable to examine the W.R. in chronic cases, I have yet to see a case in which the ætiology was syphilitic.

It is hardly likely that lupus erythematosus of the scalp could be mistaken for any luetic condition. As on the face, it is characterised by atrophy, scaling and hyperkeratosis at the mouths of the follicles affected. The slow and inevitable destruction of the follicles leads eventually to permanent baldness of a strictly localised type. In no case does L.E. affect the bone, and I have never seen frank ulceration or the crusting which a tertiary syphilis would cause.

\section{(2) The Face, including the Ears and Nose}

It is in this area that we are most often confronted by interesting problems of diagnosis. Speaking generally as regards tuberculous manifestations, young persons usually of the female sex are more prone to them than males. They are insidious in their onset and very chronic in their course. Lupus vulgaris is of course by far the commonest example of tuberculous infection in this area. It usually begins in childhood, and females are more frequently affected than males. An insidious onset and extreme chronicity, as compared with tertiary syphilis, are the rule. The latter will do more damage in a month than lupus in a year and may involve bony structures, which L.V. never does, although on the nose and ears cartilaginous structures eventually succumb. The classical "apple-jelly" nodule, when clearly demonstrable on vitropressure, is a diagnostic feature which practically settles the nature of the lesion, but it is not always apparent. Cases of lupus " tumidus" and other aberrant varieties including the "sarcoids" occur in which diascopy fails us, and in these we are thrown back on the histology and W.R. findings. I recently favoured a diagnosis of " sarcoid" (Schaumann), in a middle aged man, with subcutaneous nodular infiltration over the left side of the temporal and malar regions. But the W.R. was strongly positive, and the lesions cleared up rapidly on the usual antiluetic therapy. 


\section{TUBERCULOSIS AND SYPHILIS}

The common tendency of the lupus nodule or tubercle to recur in scars is a feature of great clinical value in the differential diagnosis from tertiary syphilis, and one which, in my opinion, overrides any other objective symptom when doubt arises. Recurrences in general are much more frequent in cutaneous tuberculosis than they are in its luetic imitators.

That cutaneous gummata could be mistaken for scrofuloderma is not outside my experience. The illustration I am circulating is an instance in point. The patient was a woman in the early forties who some weeks after the removal of alleged tuberculous glands in the neck-the quadrilateral incision is well depicted-developed infiltrations of a dusky colour mainly at the margins, and at one spot, just external to the thyroid cartilage, a small circular ulcer. The W.R. was found to be strongly positive and would have saved the patient an unnecessary operation if it had been performed when she was first seen.

Scrofuloderma is more apt to occur on the neck than on the face, for the simple reason that it is nearly always secondary to some underlying hypodermic tuberculous infection, most commonly glandular in origin. Cervical tuberculous adenitis is still unfortunately an all too common occurrence in childhood, and its co-existence or recent history afford the diagnostic clue in nearly every case of so-called scrofuloderma. When something resembling it occurs for the first time in the adult a preceeding W.R. should always anti-date any surgical procedure. In rare instances scrofuloderma, sinus formation and an ascending tuberculous lymphangitis may occur on the legs. As in the cervical type, children are more commonly the victims than adults.

A somewhat rare manifestation of lupoid type is the so-called lupus miliaris (or follicularis) facei. It usually occurs in women in the third decade and may be confused with rosacea in which, in addition to erythema, brownish papules persist on vitro-pressure. That confusion with a papular or miliary-papular syphilide might arise is conceivable, but outside my experience. In the latter disease the papules are not likely to be confined to the face. On the contrary, the chest and back are the sites of election, and the papules, distributed in clusters or groups, would not display the symmetrical arrangement 
of the tubercular lesions. Scarring in the form of small pits would tend to occur in both, but more marked in syphilis, in which, too, pigment disturbances are more apt to be associated. Laboratory assistance other than the W.R. should rarely be required.

Ulcerations of the lips, gums and buccal mucosa are an occasional source of difficulty, but this is rare nowadays owing to the Wassermann test and the rapid healing induced by one or two salvarsan injections in the venereal cases. Clinically they differ considerably too, for in tuberculosis the ulcer is usually painful and has a more eroded and frayed appearance than the clean cut or punched out characters of syphilitic destruction.

\section{T.B. Tongue Ulceration and Glossitis}

Very rarely tuberculous ulcers can also be punched out. I have under my care at the present time an elderly spinster who has attended the Royal Northern Hospital for the past twelve years for a circular indolent ulceration (rivalling that of the rodent), involving the lower lip and angle of the mouth symmetrically. If I could show her to you I doubt if anyone here would hesitate to label the deep sunken cicatrices syphilitic, yet the W.R. has always been negative, and in the course of the disease she suddenly developed a tuberculous iritis which responded exceedingly well to treatment by tuberculin injections, which also benefited the cutaneous manifestations.

\section{FOREARMS AND HANDS}

The commonest tuberculous manifestation here is the verrucose tuberculide, and both in its evolution and appearance it is so characteristic that $I$ have yet to see a case in which confusion with syphilis might arise. But it is otherwise with palmar lesions. Relatively common in the latter disease, the manifestations are scaly and circinate in type without much tendency to ulcerate, unless fissuring can be described under that heading. Tuberculides are exceedingly rare in this situation, although the verrucose variety affects the dorsum of the hands and knuckles, and is characterised by hyperkeratosis more often than by the development of the applejelly tubercle. When the warty character prevails on the 
palms or soles it is much more likely to be syphilitic than tuberculous.

So far as I am aware the nail substance is not affected by T.B. directly, while luetic ungual dystrophies and even congenital absence of the nails were by no means uncommon before the days of pre-natal anti-luetic treatment.

\section{The Trunk}

Difficulty may sometimes arise in diagnosing between tuberculides such as lichen scrofulosorum or the papulonecrotic tuberculide and the large and small papular syphilides, and it must be remembered that the former may conceivably occur in children with a positive W.R. The syphilides are usually universal and may be associated with lesions of the mucous membranes. They are split-pea in size, smooth and rotund, of a brownish-red colour and more often pigmented than their tuberculous analogues. The small papular, acuminate or miliary papular syphilide is follicular in localisation with a marked tendency to grouping-the corymbose configuration, which is highly characteristic of their ætiology. There are three main varieties of the tuberculides to be differentiated: the miliary acute cutaneous, the lichenoid, and the papulo-necrotic. The first named may be part of a generalised tuberculosis, with a high temperature and a fatal issue. Measles or some other exanthem may have preceded it, and the cutaneous lesions are pinhead to small pea-sized nodules, sometimes grouped in clusters, which, if the child survives, usually break down and ulcerate. Lichen scrofulosorum is also a disease of childhood. It is relatively benign and symmetrically distributed over the trunk and consists of small shiny papules, frequently aggregated in patches, which may be scaly and associated commonly with keratosis of the follicles. This feature not infrequently gives rise to difficulty in the diagnosis from lichen planus. The latter, however, is always intensely pruritic and never ends in pitting or other evidence of scarring. The papulonecrotic tuberculide is perhaps as frequently seen on the extensor surfaces of the limbs as on the trunk. Here again adults are rarely affected. The lesions are solitary, like the gumma, and not symmetrical as a rule. They are small and subcutaneous in origin and take a week or v.D.

$$
\text { I67 }
$$




\section{BRITISH JOURNAL OF VENEREAL DISEASES}

longer to rise to the epidermic level where they become inflamed and undergo necrosis and ulceration, which is inevitably followed by deep pitting as a more or less permanent relic in all cases. Exacerbations and relapses over some years is a common history, and these criteria, taken as a whole, are so uniform that the chances of confusion with papular syphildes or small gummata are unlikely. Errors of diagnosis between these two subcutaneous lesions do occasionally occur, however, and must have been more common before the W.R. test was available.

It is highly probable that N.A.B. injections, which are sometimes given with good results in the demonstrably tuberculous nodules, were first introduced under the misapprehension that they were subcutaneous gummata.

Special consideration of the effects of syphilis as compared with tuberculosis in the lower extremities is scarcely necessary, for there is no essential difference in type to what is seen on the hands. The only exception we must consider is erythema induratum (Bazin).

Erythema induratum, or Bazin's disease, is worthy of separate consideration in view of its relative frequency in out-patient clinics. It is a disease of young women and starts as symmetrical hypodermic nodules in the lower third of the legs. An antecedent tuberculous history, familial or personal, is not invariably obtained; but the Mantoux reaction in my experience is practically always positive, sometimes strongly so, and seems to me to present difficulties to recent attempts to explain the ætiology on circulatory deficiencies. Frequently the nodules tend to ulcerate, and when they do so the question of a luetic origin may arise. But such ulcers are characteristically seen in older women and then usually in the neighbourhood of the knee joint. They occur in the late or tertiary stages of the disease and are therefore not symmetrical, while the punched out, clean-cut edges of the cutaneous gummatous ulcer are never observed.

Histologically, Bazin's disease is mainly a fat necrosis and it is this feature that has suggested to Professor Telford and other workers the possibility of a circulatory ætiology, spastic in type, and amenable to surgical measures such as sympathectomy. Whatever the ætiology, it is undeniable that very striking cures have been attained by surgery. I am happy to record one such in I68 


\section{TUBERCULOSIS AND SYPHILIS}

my own series. The patient, an unmarried woman of twenty-six, had suffered from Bazin's disease, associated with Raynaud's syndrome and chilblains, in the upper extremities for five years. No treatment had had much effect and she was almost crippled by her symptoms. A pre-ganglionic sympathectomy, both cervicle and lumbar, was successfully carried out by Mr. Lawrence Abel, and restoration of a normal circulation with immediate healing of her ulceration and cure of the Raynaud symptoms resulted within a week.

\section{ANO-GENITAL}

It is in this region that difficulties in diagnosis most often arise, not so much between syphilis and tuberculosis, but in the differentiation of luetic from other ulcers and non-ulcerative infiltrations and granulomata, mostly contracted in coitus, either in Europe or the tropics. With these latter we are not concerned in this paper, and it is fortunate that tuberculosis here is relatively rare, at any rate, in the healthy adult. I have yet to see a primary tuberculous chancre for the reasons I have tried to explain in my introductory remarks. But if an infant is brought to you with a penile chancre or vulvar ulceration, the ætiology may conceivably be tuberculous. I would go further and say that it is more likely to be tuberculous than syphilitic.

Lupus vulgaris is rare on the genitalia but is by no means uncommon round the anus and on the buttocks, mostly in children, although of course it may have persisted since childhood into adult life. Destructive progress is not marked, far less so than on the face. Its differentiation from tertiary syphilis rarely offers any difficulty to those familiar with the clean cut, circinate lesions of cutaneous gummata. In contradistinction, the edges of a tuberculous ulcer are sloping or shelving, the base raised or nearly level with the rest of the surface and covered usually by easily bleeding, unhealthy granulations. In the neighbourhood can often be seen numbers of the characteristic "apple-jelly" nodules which are best demonstrated by vitropressure. Peri-anal sinuses are more likely to be tuberculous than luetic. But even more characteristic, practically diagnostic, is the tendency to local recurrences in scar tissue-a feature not observed in syphilis and one 


\section{BRITISH JOURNAL OF VENEREAL DISEASES}

in which the phenomena of local immunisation must be closely concerned. Cicatrisation in tuberculous cases is more likely to be followed by deformities and atresia, due to contraction of scar tissue, than in the parallel syphilitic involvement, in which specific therapy invariably leads to rapid healing with the formation of innocuous supple cicatrices.

This article and also the article Pulmonary Tuberculosis and Syphilis are based on addresses delivered before the Medical Society for the Study of Venereal Diseases, April 28th, I939. 